\title{
Hyperoxia in post-cardiac arrest: friend or foe?
}

\author{
Jean-François Llitjos, Alain Cariou \\ ${ }^{1}$ Medical Intensive Care Unit, Cochin University Hospital (APHP), Paris, France; ${ }^{2}$ Paris Descartes University, Paris, France \\ Correspondence to: Prof. Alain Cariou, MD, PhD. Medical ICU, Cochin Hospital 27 rue du Faubourg Saint-Jacques, 75014 Paris, France. \\ Email: alain.cariou@aphp.fr. \\ Provenance: This is an invited Editorial commissioned by the Section Editor Ming Zhong (Department of Critical Care Medicine, Zhongshan \\ Hospital Fudan University, Shanghai, China). \\ Comment on: Roberts BW, Kilgannon JH, Hunter BR, et al. Association Between Early Hyperoxia Exposure After Resuscitation From Cardiac Arrest \\ and Neurological Disability: Prospective Multicenter Protocol-Directed Cohort Study. Circulation 2018;137:2114-24.
}

Submitted Aug 15, 2018. Accepted for publication Sep 12, 2018.

doi: $10.21037 /$ jtd.2018.09.54

View this article at: http://dx.doi.org/10.21037/jtd.2018.09.54

Cardiac arrest (CA) is a devastating condition resulting in a high mortality rate. Even when a return of spontaneous circulation (ROSC) has been obtained, the vast majority of patients who remain comatose after a CA will subsequently die in the following days or weeks, mostly from brain injury $(1,2)$. Of importance, it has been shown that this brain injury continues even after ROSC, due to the "reperfusion injury" process. Over the recent years, intense research aiming to limit this phenomenon was performed in order to decrease the risk of brain damage after CA. However, despite encouraging preclinical results, no drug was shown to be efficient in humans $(3,4)$. To date, targeted temperature management (TTM) is the only treatment firmly recommended in comatose patients resuscitated from a CA (5).

In parallel with TTM, guidelines also suggest to prevent reperfusion injuries resulting from systemic insults, such as exposure to hyperoxia. Indeed, oxygen plays a pivotal role during cardiopulmonary resuscitation (CPR) and oxygenenriched ventilation is highly recommended during the CPR sequence. However, hyperoxia is commonly observed in resuscitated patients as a consequence of an excessive oxygen supply continued after ROSC. Over the recent years, the hypothesis of a potential deleterious effect of this postresuscitation hyperoxia has progressively emerged, based on the assumption that the increased oxygen partial pressure $\left(\mathrm{PaO}_{2}\right)$ results in overproduction of reactive oxygen species (ROS). Being highly unstable and reactive molecules, these ROS may provoke a large panel of toxic events such as DNA damages, lipid peroxidation, protein oxidation. In experimental studies, a worse neurological, histological and neurochemical outcome was reported in animals exposed to high concentration of oxygen after resuscitation (6-10). In humans, the effects of hyperoxia after CA were assessed in a randomized trial in which CA patients were randomized to either $30 \%$ or $100 \%$ inspired oxygen fraction $\left(\mathrm{FiO}_{2}\right)$ after ROSC (11). The main endpoint was the blood level of neuron-specific enolase (NSE) measured $24 \mathrm{~h}$ after resuscitation as a marker of neuronal injury. The main result was a significant increase in NSE in patients submitted to hyperoxia and not treated with therapeutic hypothermia. As an interpretation, authors hypothesized hyperoxia was probably deleterious and that therapeutic hypothermia may protect the brain from detrimental effects of hyperoxia. However, only 28 patients were included in the study, precluding from any firm conclusion. Another randomized clinical study was designed to evaluate the risk-benefit of hyperoxia in these patients, but investigators failed to safely titrate oxygen in the pre-hospital period (12). Thus, most of the available data come from retrospective studies and meta-analysis. On the whole, these human studies, which assessed the associations between hyperoxia and clinical outcomes, reported conflicting results (13-19). Evidence does not permit to know if exposure to hyperoxia contributes by itself to a worsening of reperfusion injuries or if it is only a marker of severity. At that time, current post-resuscitation guidelines recommend titrating the $\mathrm{FiO}_{2}$ in patients after CA to avoid hypoxia and prolonged exposure to hyperoxia, which is commonly defined as arterial pressure in oxygen $\left(\mathrm{PaO}_{2}\right)$ above $300 \mathrm{mmHg}$ (5) on arterial blood gas (ABG). Experts also highlighted the urgent need for well-designed prospective studies examining the effect of hyperoxia on the outcome after CA. 
In a recent issue of Circulation, Roberts et al. reported the results of a prospective multicenter cohort study evaluating the association between high oxygen tension exposure after resuscitation from CA and neurological function or death at hospital discharge, defined as a modified Rankin Scale higher than 3 (20). From July 2013 to March 2017, all adult patients successfully resuscitated from in-hospital or out-of-hospital CA and subsequently admitted within one of the 6 participating hospitals in the United States were prospectively included. All these 280 post-cardiac arrest patients underwent therapeutic hypothermia during the first 24-hour. ABG was performed one hour ( \pm 2 hours) and 6 hours ( \pm 2 hours) and hyperoxia was defined as a $\mathrm{PaO}_{2}$ higher than $300 \mathrm{mmHg}$ on one or more ABG. Regarding initial rhythm, $55 \%$ of patients had an asystole or pulseless electrical activity and 37\% had ventricular fibrillation or ventricular tachycardia (VF/ VT). Percutaneous coronary intervention was performed in $26 \%$ of patients with OHCA with VF/VT. Hyperoxia during early hours was observed in $38 \%$ of the cohort. After adjusting for potential usual baseline and postCA confounders, hyperoxia exposure was found to be an independent predictor of poor neurological function at hospital discharge ( $\mathrm{RR}=1.23,95 \% \mathrm{CI}$ : $1.11-1.35)$ using a multivariate linear regression. This finding was confirmed in multiple sensitivity analyses. In addition, authors found an association between the duration of hyperoxia exposure and neurological injury. Assuming that the $\mathrm{PaO}_{2}$ remained constant between two ABG measurements, the authors estimate the duration of exposure to hyperoxia and reported that a one-hour longer duration of hyperoxia exposure was associated with a $3 \%$ increase in the risk of poor neurological outcome ( $R R=1.03$, 95\% CI: $1.02-1.05)$. The main conclusion was that early hyperoxia exposure after resuscitation from CA was independently associated with death and poor neurological function at hospital discharge.

Authors should be congratulated for this well-conducted study that reinforced the hypothesis of hyperoxia toxicity in post-CA patients. As compared with previous clinical studies, strengths of the Robert study are multiple, including the prospective and multicentric design that allowed a powerful multivariate analysis, in addition with the multiple sensitivity analyses that confirmed the main result. Nevertheless, there are also some limitations. In addition to its purely observational design, the study suffers from the lack of mechanistic assessment that would have help to appreciate the effect of hyperoxia. In this way, it is regrettable to not have collected biomarkers of oxidative stress and antioxidant defences in order to assess the balance. Even if these biomarkers are difficult to manage ex vivo, showing that the imbalance was more pronounced in patients exposed to hyperoxia would have been an elegant way to explain the main result. In the same way, showing a higher release of brain injury biomarkers (such as NSE) in hyperoxic patients would have been useful to nourish and enhance the hypothesis. Finally, hyperoxic patients were also those with the lowest arterial pressure in $\mathrm{CO}_{2}$ (even if the difference was not significant). Considering that hypercapnia was recently shown to be associated with a better outcome in post-CA patients, one may wonder if this may or not have been associated with the difference in outcome (21).

The study from Roberts $e t$ al. highlights the necessity of well-designed randomized studies. In this way, the recently published EXACT study is illustrative of the difficulties in performing an intervention (22). In this Australian phase 2 trial, 61 patients CA patients were randomized to receive either a titrated or a liberal oxygenation through bagvalve reservoir just after ROSC. The titration of oxygen started 35 minutes after ROSC and importantly, there was only a small difference in mean oxygen saturation on arrival at emergency department between the intervention and controls (97\% vs. 99\%). No difference was observed between the 2 groups regarding outcome. Based on this feasibility trial and considering the large body of evidence coming from observational studies, the same investigators recently started a phase 3 randomised control trial (NCT3138005), aiming to compare the outcome in patients managed with two different targets of oxygen saturations (90-94\% or 98-100\%) just after CPR. Performed in several Australian cities, investigators wish to enroll nearly 1,400 OHCA patients. Survival to hospital discharge will be the primary outcome by many important secondary outcomes will be assessed such as survival at different time points, quality of life and neurological recovery, oxygen saturation values over time, and adverse events. We are eager to discover the results of this major trial that may considerably influence future guidelines and practices.

\section{Acknowledgements}

None.

\section{Footnote}

Conflicts of Interest: The authors have no conflicts of interest to declare. 


\section{References}

1. Lemiale V, Dumas F, Mongardon N, et al. Intensive care unit mortality after cardiac arrest: the relative contribution of shock and brain injury in a large cohort. Intensive Care Med 2013;39:1972-80.

2. Bougouin W, Lamhaut L, Marijon E, et al. Characteristics and prognosis of sudden cardiac death in Greater Paris: population-based approach from the Paris Sudden Death Expertise Center (Paris-SDEC). Intensive Care Med 2014;40:846-54.

3. Argaud L, Cour M, Dubien PY, et al. Effect of Cyclosporine in Nonshockable Out-of-Hospital Cardiac Arrest: The CYRUS Randomized Clinical Trial. JAMA Cardiol 2016;1:557-65.

4. Cariou A, Deye N, Vivien B, et al. Early High-Dose Erythropoietin Therapy After Out-of-Hospital Cardiac Arrest: A Multicenter, Randomized Controlled Trial. J Am Coll Cardiol 2016;68:40-9.

5. Nolan JP, Soar J, Cariou A, et al. European Resuscitation Council and European Society of Intensive Care Medicine 2015 guidelines for post-resuscitation care. Intensive Care Med 2015;41:2039-56.

6. Pilcher J, Weatherall M, Shirtcliffe P, et al. The effect of hyperoxia following cardiac arrest-a systematic review and meta-analysis of animal trials. Resuscitation 2012;83:417-22.

7. Vereczki V, Martin E, Rosenthal RE, et al. Normoxic resuscitation after cardiac arrest protects against hippocampal oxidative stress, metabolic dysfunction, and neuronal death. J Cereb Blood Flow Metab 2006;26:821-35.

8. Richards EM, Fiskum G, Rosenthal RE, et al. Hyperoxic reperfusion after global ischemia decreases hippocampal energy metabolism. Stroke 2007;38:1578-84.

9. Richards EM, Rosenthal RE, Kristian T, et al. Postischemic hyperoxia reduces hippocampal pyruvate dehydrogenase activity. Free Radic Biol Med 2006;40:1960-70.

10. Llitjos JF, Mira JP, Duranteau J, et al. Hyperoxia toxicity after cardiac arrest: What is the evidence? Ann Intensive Care 2016;6:23.

11. Kuisma M, Boyd J, Voipio V, et al. Comparison of 30 and the $100 \%$ inspired oxygen concentrations during early post-resuscitation period: a randomised controlled pilot study. Resuscitation 2006;69:199-206.

12. Young P, Bailey M, Bellomo R, et al. HyperOxic Therapy OR NormOxic Therapy after out-of-hospital cardiac arrest (HOT OR NOT): a randomised controlled feasibility trial. Resuscitation 2014;85:1686-91.

13. Kilgannon JH, Jones AE, Shapiro NI, et al. Emergency
Medicine Shock Research Network (EMShockNet)

Investigators. Association between arterial hyperoxia following resuscitation from cardiac arrest and in-hospital mortality. JAMA 2010;303:2165-71.

14. Bellomo R, Bailey M, Eastwood GM, et al. Study of Oxygen in Critical Care (SOCC) Group. Arterial hyperoxia and in-hospital mortality after resuscitation from cardiac arrest. Crit Care 2011;15:R90.

15. Helmerhorst HJ, Roos-Blom MJ, van Westerloo DJ, et al. Associations of arterial carbon dioxide and arterial oxygen concentrations with hospital mortality after resuscitation from cardiac arrest. Crit Care 2015;19:348.

16. Johnson NJ, Dodampahala K, Rosselot B, et al. The association between arterial oxygen tension and neurological outcome after cardiac arrest. Ther Hypothermia Temp Manag 2017;7:36-41.

17. Kilgannon JH, Jones AE, Parrillo JE, et al. Emergency Medicine Shock Research Network (EMShockNet) Investigators. Relationship between supranormal oxygen tension and outcome after resuscitation from cardiac arrest. Circulation 2011;123:2717-22.

18. Lee BK, Jeung KW, Lee HY, et al. Association between mean arterial blood gas tension and outcome in cardiac arrest patients treated with therapeutic hypothermia. Am J Emerg Med 2014;32:55-60.

19. Vaahersalo J, Bendel S, Reinikainen M, et al. FINNRESUSCI Study Group. Arterial blood gas tensions after resuscitation from out-of-hospital cardiac arrest: associations with long-term neurologic outcome. Crit Care Med 2014;42:1463-70.

20. Roberts BW, Kilgannon JH, Hunter BR, et al. Association Between Early Hyperoxia Exposure After Resuscitation From Cardiac Arrest and Neurological Disability: Prospective Multicenter Protocol-Directed Cohort Study. Circulation 2018;137:2114-24.

21. Eastwood GM, Schneider AG, Suzuki S, et al. Targeted therapeutic mild hypercapnia after cardiac arrest: A phase II multi-centre randomised controlled trial (the CCC trial). Resuscitation 2016;104:83-90.

22. Bray JE, Hein C, Smith K, et al. EXACT Investigators. Oxygen titration after resuscitation from out-of-hospital cardiac arrest: A multi-centre, randomised controlled pilot study (the EXACT pilot trial). Resuscitation 2018;128:211-5.

Cite this article as: Llitjos JF, Cariou A. Hyperoxia in postcardiac arrest: friend or foe? J Thorac Dis 2018;10(Suppl 33):S3908-S3910. doi: 10.21037/jtd.2018.09.54 